\begin{abstract}
Driving under the influence of alcohol is a contributing factors to a number of road traffic accidents. There is, however, a lack of research into the behaviours that lead to drink driving. The current research used a novel approach, behaviour sequence analysis, to investigate the chains of behaviours that lead to drink driving. Statements were taken from individuals $(\mathrm{N}=$ 60 ) in an interview, reporting a time they had consumed alcohol and driven a vehicle. Statements were coded and the sequences of behavioural pairs were analysed. Results were presented in a state transition diagram, and indicated a variety of behavioural sequences leading to drink driving. A significant chain of events showed that individuals who had attempted to calculate their blood alcohol concentration and then drive a vehicle felt unsafe to drive, after driving. Also, many individuals did not intend to drink and drive; however, influences such as peer pressure and being surrounded by alcohol lead to them being more likely to consume multiple alcoholic drinks, and then drive a vehicle. The current research outlines future research, and implications for polices and laws on behaviours that surround drink driving, as well as providing a new method for research.
\end{abstract}

Keywords: drink driving; laws; alcohol; behaviour sequence analysis; behaviours 


\section{Driving under the influence of alcohol: A sequence analysis approach}

Driving under the influence (DUI) of alcohol, or 'drink driving', is a significant contributor to motor vehicle accidents and crashes, leading to injuries and fatalities. In the United Kingdom, in 2013, drink driving accidents caused 260 fatalities, 1110 serious injuries, and over 8,000 casualties (Department for Transport, 2015) . While DUI rates have slowly declined in recent years, there is still a large percentage of people who drink and drive; 638,651 roadside breath tests revealed 71,675 drivers or riders who either failed or refused to take the test (Home Office National Statistics, 2015). In psychology, interventions to reduce the occurrence of drink driving typically involve a socio-cognitive approach (Jongen, Vuurman, Ramaekers, \& Vermeeren, 2016; Parker, Manstead, Stradling, \& Reason, 1992; Rowe et al., 2016). This research has been important in understanding the cognitive processes and individual differences that underpin drink driving; however, the outcome behaviour is usually constructed as an isolated event (e.g., "Driving under the influence of alcohol”), as though the outcome were a singular, isolated unit of action that occurs without need to understand surrounding behaviours or events. However, preceding behaviours or events may have a large impact on subsequent outcomes. For instance, if someone who has been drinking alcohol receives an unexpected emergency call, they may be forced into a position of needing to drive a vehicle when they normally would not. A method to explore preceding-event and subsequent-outcome relationships is clearly needed. The main goal of the current research is to fill this gap in the literature through the use of a sequence analysis approach (Bakeman \& Gottman, 1997; Bakeman \& Quera, 2011; Clarke, Forsyth, \& Wright, 1998) to understand the complex interaction between behaviours leading up to individuals driving under the influence of alcohol. 


\section{Drink driving limits and the law}

Scotland has recently reduced the legal alcohol limits for driving. The current alcohol limit for drivers in England and Wales is 80 milligrams of alcohol per 100 millilitres of blood, 35 micrograms per 100 millilitres of breath (Road Traffic Act, 1988); limits are lower in Scotland and most other European countries. While these numbers offer a threshold between legal and illegal, they are not as clear to individuals, who may assume they can consume some alcohol and drive a vehicle safely and legally (MacMillan \& Hewitt, 2008; Rowe et al., 2016; Watling \& Armstrong, 2015). Heuristics and rules-of-thumb typically lead people to believe one (or two) drinks is acceptable to consume while still being legal to drive a vehicle (Collins, Dickson, Eynon, Kinver, \& Macleod, 2008). However, the relationship between alcohol consumption and blood alcohol concentration (BAC) varies from person-toperson, based on a number of factors (e.g., weight, gender, metabolism, stress levels, diet, and type of drinker you are). While the limits for alcohol levels appear clear from a legal perspective, there is ambiguity for individuals attempting to calculate BAC based on their drinking behaviour. Research has shown that a single drink can affect cognitive abilities and reaction times (Freydier, Berthleon, Bastien-Toniazzo, \& Gineyt, 2014; Lyle Baillie International, 2005; Li et al., 2015). Therefore, the current research investigated the sequence of events that occurred leading up to an individual having a first drink of alcohol and then subsequent events that led up to the eventual driving of a vehicle.

There are a variety of preceding events that may lead up to an individual's decision to drive a vehicle while intoxicated. Therefore, psychological models that treat 'drink driving' as an isolated outcome behaviour risk reducing a complex pattern of interactions into an overly simplistic outcome. To understand the complexity of the behaviours surrounding drinking alcohol and driving a vehicle, a method is required that not only incorporates these events, but measures associations between them. For instance, understanding reasons why 
1 people either decide to drink and then drive, or decide to drink and not drive (but then have

2 plans changed) is an important factor in understanding drink driving behaviours. For

3 example, peer pressure and social dynamics have been shown to have large effects on risky

4 decision making and driving behaviours (An et al., 2013; Gheorghiu, Delhomme, \&

5 Felonneau, 2015). Therefore, research needs to incorporate the effect of peer pressure and

6 other events, on subsequent behaviours. More importantly, to be able to do this with real-

7 world data from actual events, is an important advancement in the literature, compared to

8 laboratory conditions or questionnaires. Clarke and colleagues (1998), therefore, used a

9 sequence analysis approach to understand the sequence of events leading up to vehicle

10 accidents. The current research will take a similar approach, using people's statements

11 regarding events that occurred between them consuming alcohol and driving a vehicle. This approach will elucidate key moments in which plans to not drink (or only have one drink) change into multiple drinks and later driving.

Research into driving-related accidents has previously used police records, as these are convenient and suitable for purpose (Fell, 1976; Massie, Campbell, \& Blower, 1993). There are limitations regarding this approach in relation to drink driving research. In the first instance, for a police report to be filed, the person who has consumed alcohol has to be caught, either through roadside breath-checks, or as part of a post-collision report and investigation. This leaves a large gap in the literature regarding individuals who consume alcohol and drive, but are not caught or do not end up in an accident. Therefore, attempting to understand the complex pattern of behaviours that occur in the sequence from a person's first alcoholic drink to final driving of a vehicle needs an approach beyond police records. 


\section{Sequence analysis approach}

Sequence analysis, also referred to as behaviour sequence analysis (BSA) and exploratory sequential data analysis (ESDA), is a useful method for categorising behaviours or events and investigating the sequential patterns between them (Bakeman \& Quera, 2011;

Clarke \& Crossland, 1985; Zourbanos et al., 2015). Sequence analysis shows patterns in data sequences by measuring statistical dependencies between events over the course of a particular time-period or episode (Bakeman \& Gottman, 1997; Bakeman \& Quera, 2011). In classic parametric statistics, independence of observations is a requirement; however, dependence in sequence analysis is the main focus (Bakeman \& Gottman, 1997).

Behaviour sequence analysis (BSA) is a simple method of sequence analysis for summarising complex interactions across a time period (Clarke et al., 1998; Fossi, Clarke, \& Lawrence, 2005; Lawrence, Fossi, \& Clarke, 2010). In BSA, transitions can be calculated between events that directly follow each other, or are separated by a specific number of events. In the simplest form of $\mathrm{BSA}^{1}$, the analysis determines whether given a particular antecedent event subsequent events occur more or less often than would be expected by chance. For example, in the case of driving under the influence of alcohol, sequence analysis can be used to investigate multiple transitions: does drinking a first drink (event category 'a') lead to having a second drink (event category 'b'), and does this lead to more drinks (event category ' $c$ ') $)^{2}$ eventually resulting in driving a vehicle (event category 'd'). Behaviour sequence analysis tests whether 'a-b', 'b-c', and 'c-d' pairs, for instance, occur more or less

\footnotetext{
${ }^{1}$ In the simplest form of BSA, the analysis measures the link between one antecedent event on one subsequent (sequitur) event. This can be referred to as lag one sequence analysis or first order sequence analysis. Though there are differences between lag and order at higher levels, at the level analysed in this paper, they are equivalent. Therefore, to avoid confusion, the current paper refers to the method of analysing one antecedent event on one subsequent event as 'Behaviour Sequence Analysis'.

${ }^{2}$ These may also be coded as multiple instances of a single behaviour category (i.e., 'drinks alcohol'); however, for the current research it is clearer to show the sequence of progression from the first drink, to second drink, to many drinks, rather than looping back around a single behavioural category.
} 
1 than expected by chance. This example is provided as an illustrative account, actual

2 behavioural data is typically more complex and involves more variation and transitions in

3 behaviours. Therefore, the first stage of sequence analysis is to code behaviours into mutually

4 exclusive and exhaustive categories (Bakeman \& Quera, 2011).

5

Sequence analysis has previously been used to examine the pattern of actions across a number of activities, including marital conflict (Gottman, 1979), violent episodes between people (Beale, Cox, Clarke, Lawrence, \& Leather, 1998; Turner \& Clarke, 2009), and rape cases (Fossi et al., 2005; Lawrence et al., 2010). In relation to the present research, sequence analysis has also been used to investigate road traffic accidents (Clarke et al., 1998). To the authors' knowledge, sequence analysis has not been used to study the events occurring leading up to someone deciding to drive a vehicle under the influence of alcohol. The focus of the study was on driving after consuming alcohol; this meant statements from individuals who consumed only one drink and those who consumed many drinks were included. An important reason for this is to understand whether there are distinct behavioural patterns between those who drink one or two alcoholic beverages compared to those who drink many, and then drive a vehicle. This allows a more comprehensive sequence analysis of all drinking patterns, rather than just those that occur for excessive alcohol consumption (over legal limits) and driving.

\section{Method}

\section{Participants}

A convenience sample of 60 (34 females, 26 males, $M_{\text {age }}=27, S D=12.4$, range $=18$ to 59) people participated in the current study. Participants were recruited through online media, word-of-mouth, and known contacts of the researchers. Participants classified 
1 themselves as either being University students $(n=29)$, unemployed $(n=12)$, or full-time

2 employed $(\mathrm{n}=19)$. All participants admitted to drinking at least one alcohol beverage and

3 driving a vehicle. Furthermore, all participants had been driving between 1 and 40 years $(M=$

$48.8, S D=12.1)$. Finally, all participants held a current driver's license, with between 0 and 9

5 points $(M=0.6, S D=1.9)$. All participants gave fully informed consent to participate and for

6 their data to be analysed. Participants were interviewed, one-on-one, and interviews lasted

7 approximately 15 minutes, during which they were informed about the focus of the study and

8 given time to reflect on and elaborate on their responses. The Ethics Review Board at the

9 University of [REMOVED FOR ANONYMOUS REVIEW] approved the current study.

10 Data

The data consisted of 60 statements from participants regarding a specific episode in

12 their past in which they consumed alcohol and later drove a vehicle, while still under the

13 influence of alcohol. In order to allow for more focused accounts of drink driving related

14 behaviours, participants were asked to provide a statement relating to only one particular time, rather than generalised statements. This is more analogous to police reports, which focus on a particular incident, rather than generalities. Furthermore, instructions regarding the statement were such that participants could include instances where they may have slept for a period of time, and either awoke or been awoken with the need to drive a vehicle, while still under the influence of pre-sleep drinking. Participants were instructed that their account of drink driving should be typical of their normal drink driving episodes, rather than a peculiar or out-of-the-ordinary experience. This was so that the data consisted of normal, representative drink driving episodes, rather than sequences of events that were unlikely to happen again. No participants indicated that their sequence of events were atypical in any way. 
Statements were taken from participants about their experience of consuming alcohol and driving a vehicle. Statements were selected for analysis if they met the following criteria: (a) they were detailed enough to perform BSA; (b) they outlined a single account of consuming at least one alcoholic beverage followed by subsequent behaviours and finally by driving a vehicle -clearly indicating driving occurred within the same timeframe as drinking alcohol (i.e., not enough time had lapsed for the effects of alcohol to be out of the system), (c) if they suggested they slept before driving, that the length of sleep was so short they were awoken while still feeling the influence of alcohol. Beyond these criteria, statements involving a variety of behaviours were coded.

\section{Coding scheme}

A coding scheme was formulated based on the statements given. Coding created categories that were mutually exclusive and exhaustive (Bakeman \& Quera, 2011), to ensure all important behaviours were included in analysis, without overlap or ambiguity. Coders with experience in psychological research methods and sequence analysis were selected to develop independent coding schemes and reach consensus regarding category labels. Once categories were agreed, coders were then asked to code all of the statements. Cohen's kappa was used as the preferred index for measuring interrater agreement, as it has been used previously in the literature (Cohen, 1960), and takes into account percentage of agreement above chance. Agreement scores $(k=.82)$ indicated substantial to almost perfect agreement (Landis \& Koch, 1977); therefore, the coding process was iterated until reliability was surpassed. Given the relatively straightforward nature of the statements, there were no ambiguous cases or categories, so a third rater was not required. Finally, a back-translation test, in which codes were used to recreate statements, showed that coding schemes captured 
1 the essence of statements without undue loss of important behavioural data. Regarding exact

2 number of drinks consumed, participants were encouraged to provide accurate accounts of

3 their drink driving episode; however, while all participants could accurately recall 'one' or

4 'two' drinks, participants who consumed beyond that number were less clear. Therefore, to

5 avoid confusion or ambiguous results, coders agreed that 'many drinks' was a more suitable

6 term.

\section{$7 \quad$ Statistical Analysis}

Once statements were coded into sequences of categories, data were input into the statistical package R (R Core Team, 2013), and analysed using BSA. Conditional transitional probabilities were calculated and significant transitions occurring above chance, according to chi-square statistic, were analysed. Transitions from antecedent behaviours to subsequent behaviours were presented graphically in a state transition diagram, showing the overall progression of behaviours arising from participants' statements. The state transition diagram shows only those transitions for which calculated standardised residual scores deviated significantly from their expected, or chance, values. Therefore, links represent significant relationships between events; essentially, the presence of antecedent event $A$ makes subsequent event $B$ more likely than it would have been by chance alone.

\section{Results}

The analysis of participants' statements regarding episodes in which they consumed alcohol followed sometime later by driving a vehicle, while still under the influence of alcohol, were analysed with a behaviour sequence analysis (BSA). A state transition diagram was then drawn to illustrate graphically the sequence of events in time (see Figure 1). All transitions in the diagram are significant. Standardised residuals (SRs) and frequency of 
1 transitions (in brackets) are provided alongside arrows between events ${ }^{3}$. Standardised

2 residuals provide an account of transition probabilities, accounting for base rates, and can be

3 interpreted as how likely the link between behaviours is, compared to if the behaviours

4 occurred together by chance alone. Therefore, SRs give an indication of interdependence in

5 the current data set (i.e., 'A' causes 'B' to be more likely to occur), and are of interest when

6 they reach a level above chance (Bakeman \& Gottman, 1997; Townsend et al., in press).

7 Since the SR represents the extent to which an event pair occurs significantly above what is

8 expected by chance, when that chance expectation is very low, a transition that occurs only

9 once or twice may nonetheless have a very high SR. This does not necessarily mean that the

10 transition is spurious - indeed, if event ' $\mathrm{A}$ ' only occurs twice, and both times preceding event

11 ' $\mathrm{B}$ ', this may be considered notable; but it does warrant highlighting in the results. This is

12 why the transition diagram is annotated with both SRs and observed frequencies for each

13 transition.

The first thing to note is a first-order, also known as a lag-one, BSA was used. This means only pairs of behaviours were analysed, and then high frequency pairs were placed in

16 larger chains. For example, 5 people suggested they had the pairing 'go out with friends' and

17 then 'plan not to drink'. The SR for this transition was 5, indicating a strong interdependence

18 between these behaviours. Progressing further through the state transition diagram, for

19 example, 4 people connected 'plan not to drink' to 'others drinking' (i.e., people around them

20 were drinking), which had an SR of 3.9. However, these sequences should only be read as

21 behaviour pairs (e.g., $\mathrm{A} \rightarrow \mathrm{B}$, and $\mathrm{B} \rightarrow \mathrm{C}$; where ' $\mathrm{A}$ ' is 'go out with friends', 'B' is 'plan not

22 to drink' and ' $\mathrm{C}$ ' is 'others drinking'). It may be tempting to suggest that that 9 people went

23 from 'go out with friends' via 'plan not to drink' to 'others drinking' (e.g., A $\rightarrow \mathrm{B} \rightarrow \mathrm{C}$ );

\footnotetext{
${ }^{3}$ Complete behaviour frequency data is available from the correspondence author, on request
} 
1 however, this is not the case, as only interdependence between pairs of behaviours are

2 analysed. Indeed, only 4 people suggest a pairing of 'go out with friends' and 'others

3 drinking'. Therefore, although longer chains of events can be viewed in the transition

4 diagram, they consist of significant transitions between pairs of events, rather than longer

5 chains.

6

7

8

9

\section{- Figure 1 about here -}

The state transition diagram shows a general progression of events from a starting point of people either going to a friend's house, going out with family, or friends (after finishing work in some cases), or going out with a partner. An important consideration is that there is no particular bias in final outcomes, depending on initial start points. For instance, regardless of where people start their sequences, there is no clear bias towards drink driving behaviours; the changes in behaviours towards drink driving occur later in the sequence. Also, as the diagram represents regular instances of drink driving, there are some sequences that are typical for most people in the population. For instance, several participants suggested they 'plan one drink' and then 'have first drink' $(\mathrm{SR}=7.8, \mathrm{n}=13)$. Also, in the current sample, individuals 'have first drink' and then 'eat food' $(\mathrm{SR}=10.4, \mathrm{n}=19)$. These transitions indicate many people follow their plans, and have a drink with their evening meal. These underpin the ability of BSA to reflect regular transitions. However, it is the transitions that lead to multiple alcoholic drinks, which are more important in terms of illegal drink driving behaviours.

Many participants suggested that they had originally planned not to drink alcohol.

This is where sequence analysis is most effective - in mapping the subsequent progression of events to allow an understanding of why people begin drinking after making a decision not to. For instance, after planning not to drink, several factors can influence individuals choices 
1 to then have an alcoholic drink, such as being surrounded by 'others drinking' $(\mathrm{SR}=3.9, \mathrm{n}=$

2 4), 'peer pressure' ( $\mathrm{SR}=4.2, \mathrm{n}=2)$, or changing their mind to 'plan one drink' $(\mathrm{SR}=2.2, \mathrm{n}$

3 =2). The progression from being surrounded by 'others drinking' is that some participants

4 continued to consume 'multiple drinks' themselves $(\mathrm{SR}=4.2, \mathrm{n}=7)$. Similarly, peer pressure

5 can lead to having 'multiple drinks' $(\mathrm{SR}=2.5, \mathrm{n}=2)$ or to 'having a first drink' ( $\mathrm{SR}=1.2, \mathrm{n}$

6 =2). This provides a clearer understanding of the dynamics underpinning when and how

7 people decide to begin drinking, even if they had previously decided they would not consume

8 any alcohol.

Focusing on the end of the sequence analysis, the transition between 'drive vehicle'

10 and post-drive reflections is an important finding in the current study. After participants

11 decided to, and actually did drive their vehicle, a large proportion $(\mathrm{SR}=12.2, \mathrm{n}=14)$

12 reported feeling 'not safe' and felt under the influence of alcohol. In contrast, there were

13 fewer transitions ( $\mathrm{SR}=2.1, \mathrm{n}=3$ ) between individuals who felt not safe/drunk, and then

14 decided to drive their vehicle. There were also fewer times an individual drove a car and felt

15 'in control' $(\mathrm{SR}=7.8, \mathrm{n}=9)$ compared to feelings of not being safe.

\section{Discussion}

The aim of the current study was to investigate the sequence of events that occur preceding a person drinking alcohol and then driving a vehicle. A novel method, behaviour sequence analysis, was used to show transitions between behaviours that occurred leading up to drink driving. Given the exploratory nature of the current research, no formal hypotheses were made; however, results can be shown to support some previous findings in the literature. For instance, there was evidence that some individuals decide not to drink alcohol before driving a vehicle, but through either peer pressure, environmental influence, or autonomous change of plans, they progress to drinking alcohol. This supports previous findings, which 
1 show the effect of peer pressure and environmental cues on drinking behaviours (An et al.,

2 2013; Gheorgiu et al., 2015).

A major concern surrounding drink driving laws and limits is that individuals do not actually understand or are unable to correctly calculate the correlation between what they have consumed and how that relates to legal limits (Collins et al., 2008). In the current study, some participants stated they attempted to calculate their blood alcohol concentration (BAC) and then drove a vehicle (assuming, through their calculations that they were safe to drive). For some participants this may be the case. However, several participants showed that even after one or two drinks, they went on to feel unsafe while driving. Also, no participants attempted to calculate BAC after consuming 'multiple drinks', which could mean that individuals who have consumed multiple drinks did not calculate BAC as they may have implicitly known they were over the limit, or it may simply mean that they did not even consider their BAC. It should also be noted, in support of evidence suggesting that alcohol affects individuals differently (thus compounding the problems related to attempting to calculate BAC), equal numbers of participants felt 'safe to drive as those who felt unsafe, after having a second drink. This shows the need to inform individuals more clearly about blood alcohol concentration limits and individual differences. Two drinks does not have the same effect for everyone.

The current findings also highlight the effects of alcohol on perceptions, perhaps giving individuals confidence or reducing self-assessment of risky behaviours until after the event, when realisation occurs (An et al., 2013; Gheorghiu et al., 2015). Importantly, there was no significant transition between individuals attempting to calculate their BAC and recording that they felt in control and able to drive. This may indicate that after consuming alcohol people attempt to calculate BAC and make their decision to drive based solely on this (i.e., "I'm legal, so I'm fine to drive", rather than stopping to become aware of their 
1 cognitive capabilities and feelings towards driving). This focus on the law and legality, rather

2 than practical ability is an important point in understanding individuals' decision making

3 processes. If people believe they are legally able to drive, when practically they may still be

4 impaired, this is an important consideration for drink drive limits. Indeed, as several

5 individuals did not report feeling unsafe to drive until after driving a vehicle, this could mean

6 that people are not particularly aware of their state and abilities, and only become aware

7 during or after driving a vehicle.

Possibly linked to individuals' reasoning surrounding drink driving and

9 misconceptions of how BAC works, a number of participants suggested they drank water or

10 another non-alcohol beverage (e.g., coffee) after having consumed multiple drinks.

11 Importantly, directly after consuming non-alcoholic drinks, a larger number of participants

12 suggested they then drove a vehicle. This may suggest that individuals' attempts to reduce

13 blood alcohol concentration through drinking non-alcoholic beverages may be misguided and

14 may not necessarily improve driving ability or subsequent feelings of safety. There was not a

15 significant link between consuming non-alcoholic beverages leading to feeling safe to drive a

16 vehicle.

\section{Limitations and future work}

The current data were taken from participant statements regarding their previous

19 behaviours. While this approach is similar to objective data sets, such as police reports, the

20 limitation of hindsight bias and other self-report issues persists. While this is a concern, it is a

21 limitation placed on all self-report and police statement research. The anonymous approach to

22 the current study reduced the likelihood of participants purposefully misleading researchers;

23 indeed, there is a possibility that participants in this study may have answered more openly

24 than when responding to police investigations. Future research could compare data generated 
1 by self-report statements and those generated by statements from witnesses about the same

2 episode; this would allow similarities and differences to be clearly highlighted.

The current study has shown that using BSA provides a clear account of the

4 complex, interacting events that occur around drink driving. This provides an important

5 addition to the literature as well has having large implications for future research. The

6 sequences in the current data showed similar sequences regardless of key demographics (i.e.,

7 age, gender); therefore, results may be generalizable. These findings may, therefore,

8 generalise to other countries with similar drink driving limits and laws. Given the additive

9 nature of sequence analysis, future research can be directly added to the current data set, to

10 see if differences or similarities emerge. Furthermore, future research could investigate if

11 similar sequences emerge with other drugs (i.e., cannabis). An obvious next step would be to compare the data generated by self-report statements, with police findings and records. This

13 has the benefits of furthering psychological knowledge of the sequence of behaviours involved in drink driving as well as informing police of particular behaviours to focus on when conducting investigations. For instance, police data may show different sequences or

16 patterns, which would need investigating in terms of whether suspects or witnesses are not revealing all of the information. Finally, not all of the statements collected in the current study involved illegal outcomes (e.g., speeding, accidents, being breathalysed). Clearly, contrasting sequences between those individuals who drink drive and end up in an accident or cause a fatality is an important advancement. It may be that individuals who engage in drink

21 driving resulting in fatalities have a markedly different sequence from those who do not.

22 However, while some participants suggested they only drank one alcoholic drink, research has shown that even one drink can have severe consequences of driving ability (Freydier et

24 al., 2014; Li et al., 2015); therefore, the current research may highlight those cases in which legal limits of alcohol have been consumed, but illegal outcomes occur (i.e., speeding, 
1 accidents etc.). Indeed, future research could investigate how many accidents occur for

2 individuals who have consumed within the legal limits of drink driving.

5 Sequence analysis provides a quantitative method for analysing large volumes of data. Sixty

\section{Conclusions}

The current research shows a new method for investigating drink driving behaviours. individual statements regarding drinking alcohol and driving a vehicle were analysed and result put into a clear, interpretable state transition diagram, which shows significant transitions between behaviour pairs. The results show that there is a large effect of peer pressure and social situation (i.e., others around you drinking), which may change individual's choices from not planning to drink, to consuming one or more alcohol drinks. Results also clearly underline the ambiguity individuals have regarding BAC and driving ability. After consuming two drinks, equal numbers of individuals suggested they felt safe as those who felt unsafe. Also, after driving a vehicle, more individuals admitted to feeling unsafe, than safe. These findings are useful for real world investigations into drink driving, in which a larger database could be created, allowing investigations to compare testimony with likely sequences of occurrence. This could assist with investigations into whether particular elements of a sequence need further investigation (i.e., exactly how many drinks were consumed, when, and with whom). There are also implications for policy making and interventions, in terms of using the data shown in this line of research to highlight risk sequence for drink driving. For instance, planning not to drink and drive is not enough, if you are surrounded by others drinking, which exerts a larger effect than direct peer pressure. Finally, the current research indicates that more clarity is needed for calculating BAC and understanding the effects of even one alcoholic drink on driving performance, as many individuals consumed alcohol and felt unsafe after driving a vehicle, even if they only had a 
1 couple of drinks, or attempted to calculate their BAC. This could explain why many accidents

2 occur even though individuals are within the legal limits of BAC. 


\section{References}

An, J., Sun, Y., Wang, X., Zu, P., Mai, J. C., Liang, J. P., . . Tao, F. B. (2013). [Correlation of resistance to peer pressure and risky decision-making with adolescent health risk behaviors]. Zhonghua Yu Fang Yi Xue Za Zhi, 47(3), 238-244.

Bakeman, R., \& Gottman, J. M. (1997). Observing interaction: An introduction to sequential analysis: Cambridge university press.

Bakeman, R., \& Quera, V. (2011). Sequential analysis and observational methods for the behavioral sciences: Cambridge University Press.

Beale, D., Cox, T., Clarke, D. D., Lawrence, C., \& Leather, P. (1998). Temporal architecture of violent incidents. Journal of Occupational Health Psychology, 3, 65-82.

Clarke, D. D., \& Crossland, J. (1985). Action systems: An introduction to the analysis of complex behaviour (Vol. 877): Methuen Publishing.

Clarke, D. D., Forsyth, R., \& Wright, R. (1998). Behavioural factors in accidents at road junctions: The use of a genetic algorithm to extract descriptive rules from police case files. Accident Analysis \& Prevention, 30(2), 223-234.

Cohen, J. (1960). A coefficient of agreement for nominal scales. Educational and psychological measurement, 20(1), 37-46.

Collins, E., Dickson, N., Eynon, C., Kinver, A., \& Macleod, P. (2008). Drinking and driving 2007: Prevalence, Decision making and Attitudes. Edinburgh: Scottish Government Social Research.

Department for Transport. (2015). "Estimates for reported road traffic accidents involving illegal alcohol levels: 2013”, Department for Transport, 12 February 2015 https://www.gov.uk/government/uploads/system/uploads/attachment_data/file/402698 Irrcgb-drink-drive-2013-prov.pdf.

Fell, J. C. (1976). A motor vehicle accident causal system: The human element. Human Factors, 18(85-94).

Fossi, J. J., Clarke, D. D., \& Lawrence, C. (2005). Bedroom rape sequences of sexual behavior in stranger assaults. Journal of interpersonal violence, 20(11), 1444-1466.

Freydier, C., Berthelon, C., Bastien-Toniazzo, M., \& Gineyt, G. (2014). Divided attention in young drivers under the influence of alcohol. Journal of Safety Research, 49, 1-18.

Gheorghiu, A., Delhomme, P., \& Felonneau, M. L. (2015). Peer pressure and risk taking in young drivers' speeding behavior. Transportation Research Part F: Traffic Psychology and Behaviour, 35, 101-111. doi: http://dx.doi.org/10.1016/j.trf.2015.10.014

Gottman, J. M. (1979). Marital interaction: Experimental investigations. New York: Academic Press.

Home Office National Statistics (2015). Police powers and procedures England and Wales year ending 31 March 2014. https://www.gov.uk/government/publications/policepowers-and-procedures-england-and-wales-year-ending-31-march-2014/policepowers-and-procedures-england-and-wales-year-ending-31-march-2014\#breathtests.

Summary of scientific evidence behind "Just one drink impairs driving". Report to Department of the Environment, Northern Ireland and National Safety Council, Republic of Ireland. (2005).

Jongen, S., Vuurman, E. F. P. M., Ramaekers, J. G., \& Vermeeren, A. (2016). The sensitivity of laboratory tests assessing driving related skills to dose-related impairment of alcohol: A literature review. Accident Analysis \& Prevention, 89, 31-48. doi: http://dx.doi.org/10.1016/j.aap.2016.01.001 
Landis, J. R., \& Koch, C. G. (1977). The measurement of observer agreement for categorical data. Biometrics, 33(159-174).

Lawrence, C., Fossi, J., \& Clarke, D. (2010). A sequential examination of offenders' verbal strategies during stranger rapes: the influence of location. Psychology, Crime \& Law, $16(5), 381-400$.

Li, Z., Jin, X., \& Zhao, X. (2015). Drunk driving detection based on classification of multivariate time series. Journal of Safety Research, 54, 61.e29-64. doi: http://dx.doi.org/10.1016/j.jsr.2015.06.007

Lyle Baillie International (2005). Summary of scientific evidence behind "Just one drink impairs driving'. Report to Department of the Environment, Northern Ireland and National Safety Council, Republic of Ireland.

MacMillan, K., \& Hewitt, E. (2008) Qualitative research with young people: Road Safety. Scottish Government Social Research.

Massie, D. L., Campbell, K. L., \& Blower, D. F. (1993). Development of a collison typology for evaluation of collision avoidance strategies. Accident Analysis \& Prevention, 25(241-257).

Parker, D., Manstead, A. S. R., Stradling, S. G., \& Reason, J. T. (1992). Determinants of intention to commit driving violations. Accident Analysis \& Prevention, 24(2), 117 131. doi: http://dx.doi.org/10.1016/0001-4575(92)90028-H

R Core Team. (2013). $R$ : A language and environment for statistical computing. $R$ Foundation for Statistical Computing, Vienne, Austria. ISBN 3-900051-07-0, URL http://www.R-project.org/.

Rowe, R., Andrews, E., Harris, P. R., Armitage, C. J., McKenna, F. P., \& Norman, P. (2016). Identifying beliefs underlying pre-drivers' intentions to take risks: An application of the Theory of Planned Behaviour. Accident Analysis \& Prevention, 89, 49-56. doi: http://dx.doi.org/10.1016/j.aap.2015.12.024

Townsend, E. Wadman, R., Sayal, K., Armstrong, M., Harroe, C., Majumder, P., Vostanis, P., \& Clarke, D. D. (in press). Uncovering key patterns in self-harm in adolescents: Sequence analysis using the Card Sort Task for Self-Harm (CaTS). Journal of Affective Disorders.

Turner, K., \& Clarke, D. D. (2009). Aggression in Intellectual Disability - A New Approach. Mental Health Review Journal, 14(2), 28-36. doi: doi:10.1108/13619322200900012

Watling, H., \& Armstrong, K. (2015). Exploring the influences of country-level factors on mature-aged women's drink driving attitudes. Transportation Research Part F:

Traffic Psychology and Behaviour, 30, 57-65. doi: http://dx.doi.org/10.1016/j.trf.2015.02.001

Zourbanos, N., Tzioumakis, Y., Araújo, D., Kalaroglou, S., Hatzigeorgiadis, A., Papaioannou, A., \& Theodorakis, Y. (2015). The intricacies of verbalizations, gestures, and game outcome using sequential analysis. Psychology of Sport and Exercise, 18, 32-41. doi: http://dx.doi.org/10.1016/j.psychsport.2014.12.003 